\title{
Proteomic characterization of a temperature-sensitive conditional lethal in Drosophila melanogaster
}

\author{
KS Pedersen ${ }^{1,2}$, MC Codrea ${ }^{3}$, CJ Vermeulen ${ }^{1,4}$, V Loeschcke ${ }^{1}$ and E Bendixen ${ }^{3}$ \\ ${ }^{1}$ Department of Biological Sciences, Aarhus University, Aarhus C, Denmark; ${ }^{2}$ Department of Genetics and Biotechnology, Aarhus \\ University, Tjele, Denmark and ${ }^{3}$ Department of Animal Health, Welfare and Nutrition, Aarhus University, Tjele, Denmark
}

\begin{abstract}
Genetic variation that is expressed only under specific environmental conditions can contribute to additional adverse effects of inbreeding if environmental conditions change. We present a proteomic characterization of a conditional lethal found in an inbred line of Drosophila melanogaster. The lethal effect is apparent as a large increase in early mortality at the restrictive temperature $\left(29^{\circ} \mathrm{C}\right)$ as opposed to normal survival at the permissive temperature $\left(20^{\circ} \mathrm{C}\right)$. The increased mortality in response to the restrictive temperature is probably caused by a single recessive major locus. A quantitative trait locus (QTL) region segregating variation affecting the lethal effect has been identified, allowing for a separation of primary/causal effects and secondary consequences in the proteome expression patterns observed. In this study, the proteomic response to the restrictive temperature in the lethal-line (L-line) was compared with the response in an inbred-control-line (IC-line) and an outbred-control-line (OC-line). Quantitative
\end{abstract}

protein changes were detected using isobaric tags for relative and absolute quantitation (iTRAQ) two-dimensional liquid chromatography-tandem mass spectrometry. In all, 45 proteins were found to be significantly differently regulated in response to the restrictive temperature in the L-line as compared with the IC-line. No proteins were significantly differently regulated between the IC-line and the OC-line, verifying that differential protein regulation was specific to a genetic defect in the L-line. Proteins associated with oxidative phosphorylation and mitochondria were significantly overrepresented within the list of differentially expressed proteins. Proteins related to muscle contraction were also found to be differentially expressed in the L-line in response to the restrictive temperature, supporting phenotypic observations of moribund muscle hypercontraction.

Heredity (2010) 104, 125-134; doi:10.1038/hdy.2009.132; published online 7 October 2009

Keywords: quantitative proteomics; drosophila; conditional lethal; mitochondrial dysfunction; inbreeding depression; cryptic variation

\section{Introduction}

Even though fitness reductions as a consequence of inbreeding have been known for centuries, there is still considerable debate concerning the genetic architecture of inbreeding depression and the contribution of major genes (for example, lethals) to the inbreeding load (Charlesworth and Charlesworth, 1999). Understanding the genetic basis of inbreeding depression is, therefore, crucial for evaluating the evolutionary consequences of inbreeding. Recent evidence has shown that a large amount of inbreeding depression is caused by complex epistatic interactions between genes (Ayroles et al., 2009). This indicates that inbreeding depression is caused by a few deleterious genes with major effect that, in turn, affect a large proportion of the rest of the genome (Ayroles et al., 2009). Thus, to dissect the genetic basis of inbreeding depression, it is essential to separate observed molecular responses to inbreeding into causal

Correspondence: Dr KS Pedersen, Faculty of Agricultural Sciences, Department of Genetics and Biotechnology, Blichersallé 20, Tjele, DK8830, Denmark.

E-mail: kamillas.pedersen@agrsci.dk

${ }^{4}$ Current address: Evolutionary Genetics, Centre for Ecological and Evolutionary Studies, University of Groningen, Haren, The Netherlands. Received 26 January 2009; revised 4 August 2009; accepted 3 September 2009; published online 7 October 2009 effects and secondary consequences. Moreover, genetic buffer mechanisms that counterbalance genetic defects at optimal conditions are thought to be less effective under stressful conditions, which can result in expression of cryptic genetic variation (Rutherford and Lindquist, 1998; Maisnier-Patin et al., 2005; Bobula et al., 2006). Expression of this cryptic deleterious genetic variation can partly explain the observation that inbreeding depression is often more severe under specific and more stressful environmental conditions (Armbruster and Reed, 2005). An important consequence of conditional expression of the genetic load is that purging of deleterious genetic variation is very inefficient when the trait is expressed only under specific environmental conditions. As such, populations can accumulate high levels of conditional deleterious variation under benign conditions, making them highly sensitive to environmental change, and thereby pose a threat to the longterm survival of the population.

Analyses of genetic variation that are expressed only under specific environmental conditions permit a detailed functional analysis of major genetic defects that are otherwise difficult to study (Jedlicka et al., 1997; Willis et al., 2006). Environmentally conditioned mutations covering the whole genome can easily be obtained; in particular, the fruitfly (Drosophila melanogaster) has been used extensively as a model organism in this context (Suzuki, 1970; Tasaka and Suzuki, 1973; Arking, 1975; 
Mitchell and Petersen, 1982; Homyk et al., 1986). Detailed genetic and molecular studies of relatively simple conditionally expressed genetic defects can contribute to an improved understanding of the causations of inbreeding depression, and identify the cascades of processes affected by expression of a single or few deleterious mutations with major fitness effects. Analyses of simple genetic defects are of general relevance for improving knowledge on how the expression of major genetic effects (for example, inheritable syndromes and diseases) influences post-transcriptional molecular levels, that is, to separate cause and consequences. Quantitative trait locus (QTL), microarray and proteome analyses enable us to study the consequences of inbreeding from different perspectives. Although integration of the results obtained from the different molecular techniques is still far from straightforward, a combination of these approaches will undoubtedly provide new exiting insights into the molecular basis of inbreeding depression and the causation of genetic load.

Proteome analysis is particularly well suited to characterize population-specific differences on a biological level very close to phenotypic fitness (Chevalier et al., 2004; Biron et al., 2006; Karr, 2008). In particular, genotypeenvironment interactions might be regulated by changes in cis/trans regulating factors, methylation status and post-translational modifications. Proteome analysis can be thought of as a phenotypic characterization with the potential to distinguish different genotypes at the biochemical level, thereby extending classic genetic research (Thiellement et al., 2002; Chevalier et al., 2004). Integrated interpretation of results from classic genetic analysis, microarray data and proteome data have previously been used to characterize the integrated whole-organism response, and consequently identify the causal mutation of the lethal phenotype (Thomsen et al., 2006).

A temperature-induced conditional lethal in an inbred line of $D$. melanogaster was reported by Vermeulen and Bijlsma (2004a, b). The lethal allele was discovered as a consequence of an inbreeding procedure, indicating that the lethal effect was caused by a naturally occurring deleterious allele and may thus be associated with conditional inbreeding depression in natural populations. The conditional lethality is temporally stable indicating a fixation of the lethal allele in the population. To obtain insight into the physiological processes disturbed by expression of the lethal allele and potentially pinpoint the dysfunctional process causing the lethal effect, we analyzed the environmentally induced proteome changes specific to the lethal-line (L-line; inbreeding coefficient $\approx 0.95$, line LI4 in Vermeulen and Bijlsma, 2004a). An explorative proteome analysis can significantly shorten the list of causal candidate genes underlying the lethal effect. Maintenance of L-line adult flies at the permissive temperature $\left(20^{\circ} \mathrm{C}\right)$ causes normal adult survival, whereas adult mortality at the restrictive temperature $\left(29^{\circ} \mathrm{C}\right)$ is extremely high (Vermeulen and Bijlsma, 2004b). A randomly chosen inbred line (IC-line; inbreeding coefficient $\approx 0.95$, line CI13 in Vermeulen and Bijlsma, 2004a) and an outbred line (OC-line; inbreeding coefficient $=0$, line O6 in Vermeulen and Bijlsma 2004a), showing normal adult viability, were chosen as control lines (for details see Vermeulen and Bijlsma, 2004b). All lines were established in 1997 from the same base stock population (G83; see Bijlsma et al., 2000 for details) and the lethal effect was first showed in 1999 (Bijlsma et al., 2000). The lethal effect is suggested by QTL mapping and previous genetic experiments to be caused primarily by a recessive major single locus (Vermeulen and Bijlsma, 2004b; Vermeulen et al., 2008). The QTL region, harboring a mutation influencing mortality at the restrictive temperature, is positioned at the left arm of the second chromosome (Vermeulen et al., 2008). The region spans from cytological position 30F-35B, with a peak at 33C-E. The second chromosome in $D$. melanogaster has previously been estimated to have a high potential for heat-sensitive mutations (Mayoh and Suzuki, 1973; Tasaka and Suzuki, 1973). The lethal effect was first found to be restricted to male flies, but subsequent QTL analysis identified segregating genetic variation for the lethal effect in females. In addition to temperature-specific expression, sex-specific expression could thus also be a mechanism by which the lethal mutation has sustained segregation in the population.

The aim of this study was to characterize proteome changes induced by expression of a conditional lethal mutation found in an inbred line of D. melanogaster. A characterization of the environmentally induced proteome changes specific to the L-line will provide insight into the molecular processes causing high mortality in response to the restrictive temperature in the L-line. Characterization of the cascade of processes that are affected by expression of the lethal mutation can give an indication of which processes to search for the causal mutation. The cytological positions of genes coding for the proteins observed to be differentially regulated in the L-line are matched to the QTL region (30F-35B). Matching of proteome expression patterns to the QTL region provides a unique opportunity to separate primary causes and secondary effects.

\section{Materials and methods}

\section{Experimental design}

An overview of the experimental design is shown in Figure 1. Proteome analysis was performed in three independent experiments. In experiment 1 , the response to the restrictive temperature in the L-line was compared with the response in the IC-line. To identify differences between the L-line and the IC-line that could be due to general inbreeding effects in the IC-line, the IC-line was compared with an outbred control (OC-line) in experiment 2. Experiment 3 was accomplished approximately 40 generations after experiments 1 and 2 to validate the general and major proteome changes observed in experiment 1. Protein extraction, digestion and labelling were replicated twice for experiments 1 and 2 to account for differences caused by experimental variation (Gan et al., 2007). For experiment 3, protein extraction, digestion and labelling was performed once. Mass spectrometry analysis of each four-plex sample was performed twice to increase protein coverage and confidence (Chong et al., 2006). Data from all mass spectrometry analyses within each replicate were searched together in ProteinPilot 1.0 (Applied Biosystems, Foster City, CA, USA).

\section{Expression of lethal effect}

Adult flies from the three different lines (L-line, IC-line and OC-line) were kept in bottles with fresh standard medium and live yeast at $20^{\circ} \mathrm{C}$ for 3 days. For each line, 


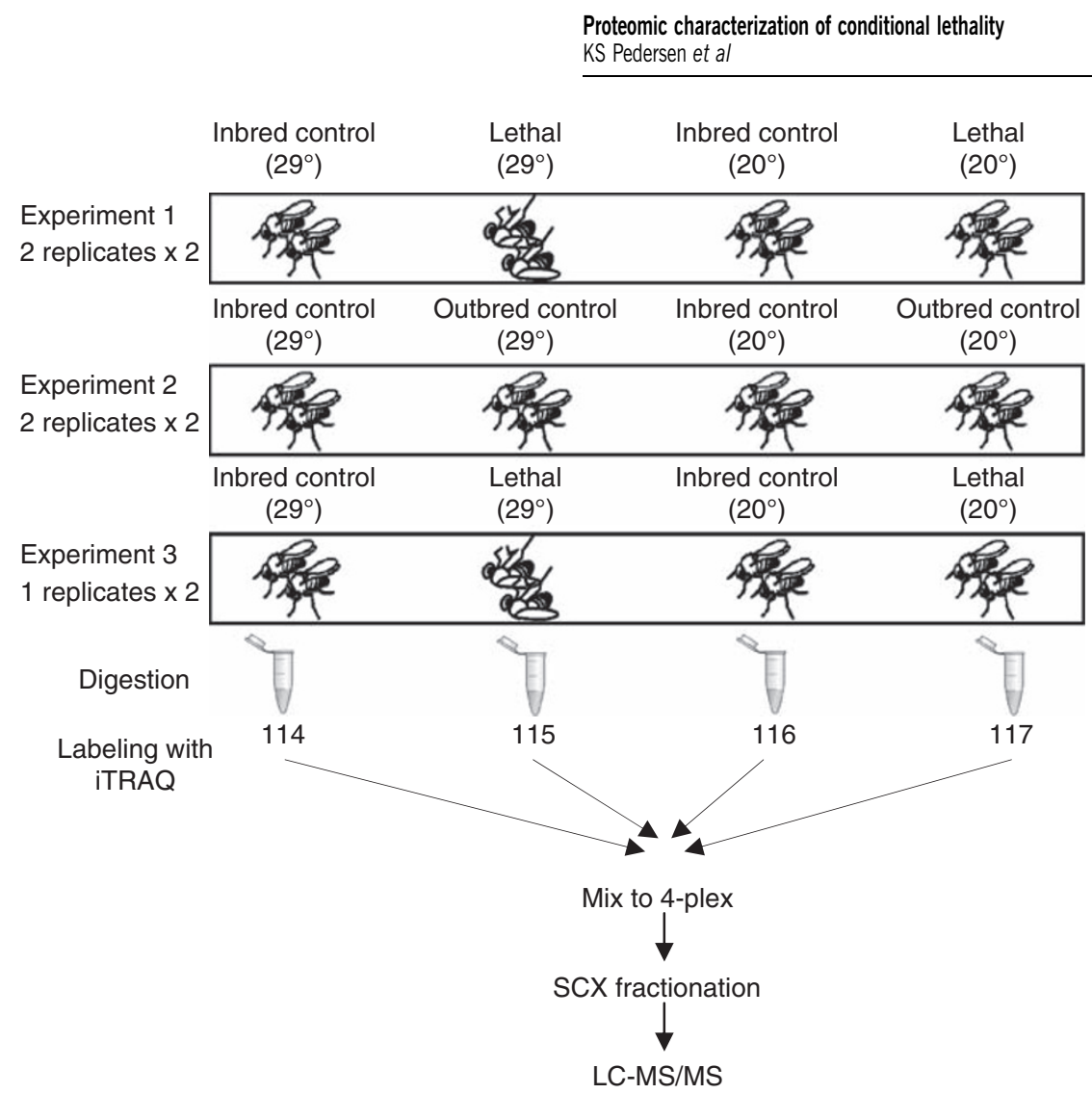

Figure 1 Experiment 1 compares the lethal-line (L-line) and inbred-control-line (IC-line) and experiment 2 compares the IC-line and outbredcontrol-line (OC-line). The flies in experiments 1 and 2 are sampled in the same generation. Experiment 3 compares the L-line and IC-line. Flies for experiment 3 are sampled approximately 40 generations after experiments 1 and 2. Proteins are digested by trypsin, labelled with isobaric tags for relative and absolute quantitation (iTRAQ) allowing for relative quantification, mixed to a four-plex, strong cation exchange fractionated and separated on an online LC-column before tandem mass spectrometry.

four females and one male were collected and transferred into each of 40 vials and allowed to oviposit for 2 days at $25^{\circ} \mathrm{C}$. A low density was ensured in the bottles and the flies developed at $25^{\circ} \mathrm{C}$. Male flies $(0-48 \mathrm{~h})$ were collected into vials with fresh medium. In all, 32 vials with 20 males were collected for each line. Half of them were kept at $20^{\circ} \mathrm{C}$ and the other half at $29^{\circ} \mathrm{C}$. Flies were transferred to new vials with fresh medium at day 3 . At day 4 , at the onset of the lethal phase, they were killed and snap-frozen in liquid nitrogen and stored at $-80^{\circ} \mathrm{C}$. No dead flies were sampled (mortality until sampling is shown in Figure 2). The remaining flies were used to estimate expression of the lethal effect (Figure 2). The protocol used for all three experiments was the same.

\section{Sample preparation}

From each treatment and line, $100 \mathrm{mg}$ male flies (approximately 150 individuals) were mechanically homogenized in $1 \mathrm{ml}$ TES buffer $(10 \mathrm{mM}$ Tris- $\mathrm{HCl}, \mathrm{pH}$ 7.6; $1 \mathrm{mM}$ EDTA; and $0.25 \mathrm{M}$ sucrose) using a Qiagen Tissuelyser (QIAGEN, Copenhagen, Denmark). Pooling of flies decreases the effects of individual variation and strengthens the tandem mass spectrometry analysis, thereby increasing the chance of detecting general regulation patterns distinct to the L-line. Samples were centrifuged for $30 \mathrm{~min}$ at $4{ }^{\circ} \mathrm{C}$ at $10000 \mathrm{~g}$ and the supernatant was frozen at $-80^{\circ} \mathrm{C}$. Overall protein concentration was estimated using Pierce bicinchoninic acid protein assay kit (Bie and Berntsen, Rodovre, Denmark) with bovine serum albumin as a standard. All buffers for

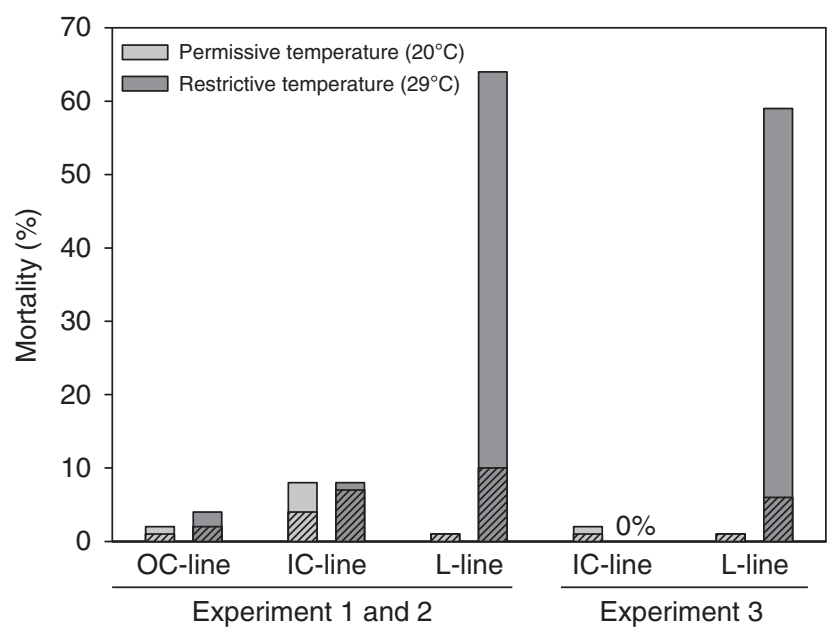

Figure 2 Mortality in response to the permissive and restrictive temperatures in the outbred-control-line (OC-line), inbred-controlline (IC-line) and lethal-line (L-line). Hatched bars indicate mortality from eclosion until day 4 (sampling) and grey bars indicate additional mortality from day 4 until day 12 .

digestion and label reagents were obtained from the isobaric tags for relative and absolute quantitation (iTRAQ) Multiplex kit (Applied Biosystems). Protein digestion and labelling of peptides with the four isobaric tags were performed according to the manufacturer's manual. Protein $(100 \mu \mathrm{g})$ was precipitated using ice-cold acetone in a 1:6 ratio $\left(\mathrm{vv}^{-1}\right)$ and re-suspended in $20 \mu \mathrm{l}$ 
dissolution buffer ( $0.5 \mathrm{M}$ triethyl-ammonium-bicarbonate and $0.1 \% \mathrm{wv}^{-1}$ sodium dodecyl sulfate). Cysteine residues were reduced using $2.5 \mathrm{mM}$ Tris-(2-carboxyethyl)-phosphine for $1 \mathrm{~h}$ at $60^{\circ} \mathrm{C}$ and then blocked for $10 \mathrm{~min}$ at room temperature using $10 \mathrm{~mm}$ methylmethanethiosulfate. Samples were digested with trypsin (Applied Biosystems) overnight at $37^{\circ} \mathrm{C}$. Labelling with isobaric tags was carried out by adding the specific iTRAQ reagents ( 1 unit in $70 \mu$ l ethanol) and incubating for $1 \mathrm{~h}$ at room temperature. The samples were labelled with the isobaric tags (114-117) according to Figure 1. The four differently labelled samples were mixed in a four-plex in a 1:1:1:1 ratio and filtered through a $0.2-\mu \mathrm{m}$ centrifuge filter (National Scientific Company, Rockwood, TN, USA) by centrifugation for $10 \mathrm{~min}$ at $10600 \mathrm{~g}$. The four-plex sample was divided in samples ready for high performance liquid chromatography (HPLC) analysis, freeze dried and frozen at $-80^{\circ} \mathrm{C}$.

\section{Strong cation exchange fractionation and liquid chromatography-tandem mass spectrometry}

Peptide separation and mass spectrometry analyses were performed as previously described (Danielsen et al., 2007). Essentially, the labelled peptides were re-suspended in $0.03 \%\left(\mathrm{v} \mathrm{v}^{-1}\right)$ formic acid, $5 \%\left(\mathrm{v} \mathrm{v}^{-1}\right)$ acetonitrile in water, and $55 \mu \mathrm{g}$ of peptide mixture was loaded on an Agilent 1100 Series capillary HPLC with a Zorbax Bio-Strong Cation Exchange Series II, $0.8 \times 50 \mathrm{~mm}$ column with a flow rate of $15 \mu 1 \mathrm{~min}^{-1}$ (Agilent Technologies, Palo Alto, CA, USA). A gradient of increasing concentrations of $\mathrm{NaCl}$ was used to elute the peptides (0-3 min 0\% B, 7 min 1.5\% B, 12 min $4 \%$ B, 25 min $10 \%$ B, $35 \mathrm{~min} 50 \% \mathrm{~B}$ and $61 \mathrm{~min} 100 \% \mathrm{~B}$ ). Buffer A contained $0.03 \%\left(\mathrm{v} \mathrm{v}^{-1}\right)$ formic acid and $5 \%\left(\mathrm{v} \mathrm{v}^{-1}\right)$ acetonitrile in water and buffer B contained $0.03 \%\left(\mathrm{v} \mathrm{v}^{-1}\right)$ formic acid, $5 \%\left(\mathrm{v} \mathrm{v}^{-1}\right)$ acetonitrile and $1 \mathrm{M} \mathrm{NaCl}$ in water. Eluted peptides were pooled into 12 fractions for further analysis. Before tandem mass spectrometry analysis, the 12 fractions were further separated on an Agilent 1100 Series nano-flow HPLC (Agilent Technologies). To ensure sample desalting and fast sample loading, the HPLC system was equipped with an isocratic pump, working at $20 \mu \mathrm{lmin}^{-1}$, to load the sample on an enrichment column (Zorbax 300SB C18, $0.3 \times 5 \mathrm{~mm}$, $5 \mu \mathrm{m}$ particle). Subsequently, the enrichment column was switched into the nano-flow path $\left(300 \mathrm{nl} \mathrm{min}^{-1}\right)$ and the peptides were eluted and separated on an analytical column (Zorbax 300SB C18, $75 \times 150 \mathrm{~mm}, 3.5$ particles) with a gradient of organic solvent (0-7 min 5\% B, $70 \mathrm{~min} 40 \%$ B, $73 \mathrm{~min} 95 \%$ B, $78 \mathrm{~min} 95 \%$ B, $83 \mathrm{~min} 5 \%$ $\mathrm{B}$ and $100 \mathrm{~min} 5 \% \mathrm{~B})$. Buffer A contained $5 \%\left(\mathrm{vv}^{-1}\right)$ acetonitrile and $0.1 \%\left(\mathrm{v} \mathrm{v}^{-1}\right)$ formic acid in water and buffer B contained 5\% $\left(\mathrm{v} \mathrm{v}^{-1}\right)$ water and $0.1 \%\left(\mathrm{v} \mathrm{v}^{-1}\right)$ formic acid in acetonitrile. The HPLC system was directly connected with the mass spectrometer and the eluting peptides were sprayed through a nanospray needle (Fused Silica Emitters, OD $360 \mu \mathrm{m}$, ID $75 \mu \mathrm{m}$, Proxeon Biosystems, Odense, Denmark) into the Q-star Elite mass spectrometer (Applied Biosystems).

\section{Protein identification and quantification}

Proteins were identified and quantified using ProteinPilot 1.0 (Applied Biosystems) searching the Drosophila database (KBMS5.020050302) from Celera Discovery
Systems (Applied Biosystems). False positives in species-specific searches were avoided by including a contaminant database in the search process, as recommended by the manufacturer. Relative protein quantification was estimated using the Paragon Algorithm (Applied Biosystems) (Shilov et al., 2007). The results were further processed with the Pro Group Algorithm (Applied Biosystems) identifying the minimal set of justifiable identified proteins. An Unused ProtScore of 1.3 was used as a threshold, corresponding to an identification confidence of 95\%. The Unused ProtScore estimates the confidence by which a protein can be validated, using only peptide data that are not better explained by a higher ranking protein match. The following searching parameters were used: trypsin digestion, terminal, $\mathrm{K}$ and $\mathrm{Y}$ iTRAQ labels, methylmethanethiosulfate-labelled cysteine, and allows for more than 80 variable modifications. All average ratios for proteins and peptides were corrected for bias within each replicate, compensating for pipetting errors during labelling. Bias correction is based on the assumption that the majority of proteins are not differentially expressed. Identified proteins from the two replicates within each experiment were merged. Proteins were grouped using both Panther ID and SwissProt accession number to ensure correct grouping of identical proteins. Only proteins that were observed and quantified in both replicates were included in further data analysis.

\section{Statistical analysis}

For experiments 1 and 2, peptide information for all identified and quantified proteins was extracted, and bias-corrected peptide ratios were used as replicate measures of protein quantification. Calculation of average relative protein ratios from the replicates within each experiment were performed using the statistical methods of ProteinPilot 1.0. Only unique prototypic peptides were included for quantification of proteins. Excluding shared peptides served to eliminate the possibility of false masking of changes in expression. The aim was to identify proteins that respond significantly differently to the restrictive temperature in the L-line as compared with the IC and the OC-lines. Translated into statistical terms, proteins responding differently to the restrictive temperature were proteins showing a significant interaction effect between line and temperature. In experiment 1 , for each protein the response to the restrictive temperature in the IC-line was compared with the response in the L-line. Similarly, for experiment 2, for each protein the response to the restricted temperature in the IC-line was compared with the response in the OC-line. Experiment 2 was performed to identify general inbreeding effects in the IC-line that could affect interpretation of the results from experiment 1 . By testing the response in the L-line against the response in the IC-line, we identified proteins that responded differently to the restrictive temperature in the L-line. This ignores differences between the L-line and the IC-line that are temperature independent. The proteins differentially regulated in response to the restrictive temperature were most likely related with the expression of the conditional lethality in the L-line. A paired $t$-test was performed for each protein using the bias-corrected peptide ratios as the variable effect, testing for a differential regulation in 
response to the restrictive temperature. Using the biascorrected peptide ratios for each protein as the dependent variable in a paired $t$-test is a very conservative approach. Only tests on proteins that are identified and quantified numerous times (with enough statistical power) have the potential to reject the $\mathrm{H}_{0}$ hypothesis, which is that the lines respond similar to the restrictive temperature. Correction for multiple testing was performed using a method suggested by Storey (2007), which previously has been shown to be superior in performance compared with other multiple testing procedures. This method uses all relevant information across tests to calculate the significance of each test. Very briefly, the power of the method is that it maximizes the total number of expected true positives for each number of expected false positives. The lower replication in experiment 3 does not allow a test of differential regulation pattern in response to the restrictive temperature; thus, in experiment 3 protein expression differences between the L-line and the IC-line are identified within temperature regime directly using the ProteinPilot 1.0 output. Each experiment was treated statistically as independent.

\section{Results}

We identified and quantified approximately 500 proteins within each replicate with high confidence and coverage in spite of the high sample complexity due to the use of whole-fly extracts (for all experiments, the average unused ProtScore $=9.2$ and average protein sequence coverage $=39 \%$; see Supplementary Figures S1 and S2). In all, 45 proteins were found to be significantly differently regulated in response to the restrictive temperature in the L-line as compared with the IC-line, suggesting that these proteins are related to expression of the conditional mortality in the L-line. Proteins signifi-

Table 1 A list of differentially regulated proteins in response to the restrictive temperature in the L-line

\begin{tabular}{|c|c|c|c|c|c|}
\hline Protein name & FlyBase ID & $O x$ & $M$ & P-value & Cytological position \\
\hline Transferrin 1 & FBgn0022355 & & & $2.15 \mathrm{E}-28$ & 17A9-17A9 \\
\hline Adh related & FBgn0000056 & & & $1.08 \mathrm{E}-22$ & 35B3-35B3 \\
\hline Sarcoplasmic calcium-binding protein 1 & FBgn0020908 & & & $8.78 \mathrm{E}-10$ & $80 \mathrm{~A}-80 \mathrm{~B}$ \\
\hline Tropomyosin 1 & FBgn0003721 & & & $1.91 \mathrm{E}-09$ & 88E12-88E13 \\
\hline Tropomyosin 2 & FBgn0004117 & & & $2.18 \mathrm{E}-08$ & 88E13-88E13 \\
\hline ATP synthase, subunit $d$ & FBgn0016120 & ox & $\mathrm{m}$ & $3.16 \mathrm{E}-08$ & 91F1-91F1 \\
\hline Myosin heavy chain & FBgn0086783 & & & $6.61 \mathrm{E}-08$ & 36B1-36B1 \\
\hline Phosphoglyceromutase & FBgn0014869 & & & $2.14 \mathrm{E}-07$ & 98F12-98F13 \\
\hline Stress-sensitive B & FBgn0003360 & & $\mathrm{m}$ & $3.83 \mathrm{E}-07$ & 9E10-9F1 \\
\hline ATP synthase- $\beta$ & FBgn0010217 & ox & $\mathrm{m}$ & $6.97 \mathrm{E}-07$ & 102D1-102D1 \\
\hline Cytochrome P450 4g1 & FBgn0010019 & & & $9.50 \mathrm{E}-07$ & 1B4-1B4 \\
\hline CG11963 & FBgn0037643 & & & $5.91 \mathrm{E}-06$ & 85B7-85B7 \\
\hline CG3560 & FBgn0030733 & ox & $\mathrm{m}$ & $6.57 \mathrm{E}-06$ & 14B8-14B8 \\
\hline Isocitrate dehydrogenase & FBgn0001248 & & $\mathrm{m}$ & $1.95 \mathrm{E}-05$ & $66 C 8-66 C 8$ \\
\hline CG8036 & FBgn0037607 & & & $2.67 \mathrm{E}-05$ & 85A5-85A5 \\
\hline CG3192 & FBgn0029888 & ox & $\mathrm{m}$ & $4.40 \mathrm{E}-05$ & $6 C 5-6 C 5$ \\
\hline CG1516 & FBgn0027580 & & $\mathrm{m}$ & $8.15 \mathrm{E}-05$ & 46B4-46B4 \\
\hline Porin & FBgn0004363 & & $\mathbf{m}$ & $1.35 \mathrm{E}-04$ & 32B1-32B1 \\
\hline Obp44a & FBgn0033268 & & & $2.56 \mathrm{E}-04$ & 44B3-44B3 \\
\hline Myosin light chain alkali & FBgn0002772 & & & $4.11 \mathrm{E}-04$ & 98A14-98A15 \\
\hline Larval visceral protein $\mathrm{H}$ & FBgn0002570 & & & $4.70 \mathrm{E}-04$ & 44D1-44D1 \\
\hline CG9090 & FBgn0034497 & & $\mathrm{m}$ & $5.27 \mathrm{E}-04$ & 56F15-56F16 \\
\hline CG4769 & FBgn0035600 & ox & $\mathrm{m}$ & $5.31 \mathrm{E}-04$ & $64 C 13-64 C 13$ \\
\hline Stress-inducible humoral factor Turandot A & FBgn0028396 & & & $1.12 \mathrm{E}-03$ & $93 \mathrm{~A} 2-93 \mathrm{~A} 2$ \\
\hline Esterase 6 precursor & FBgn0000592 & & & $1.20 \mathrm{E}-03$ & 69A1-69A1 \\
\hline CG9029 & FBgn0031746 & & & $1.38 \mathrm{E}-03$ & 26A1-26A1 \\
\hline CG9468 & FBgn0032069 & & & $1.68 \mathrm{E}-03$ & 29F1-29F1 \\
\hline CG3321 & FBgn0038224 & ox & $\mathrm{m}$ & $2.38 \mathrm{E}-03$ & 88B4-88B4 \\
\hline CG18815 & FBgn0042138 & & & $2.73 \mathrm{E}-03$ & 68D1-68D1 \\
\hline Senescence marker protein-30 & FBgn0038257 & & & $3.27 \mathrm{E}-03$ & 88D2-88D2 \\
\hline CG3731 & FBgn0038271 & ox & $\mathrm{m}$ & $3.28 \mathrm{E}-03$ & 88D6-88D6 \\
\hline Accessory gland-specific peptide $26 \mathrm{Aa}$ & FBgn0002855 & & & $3.66 \mathrm{E}-03$ & 26A1-26A1 \\
\hline Probable phosphoserine aminotransferase & FBgn0014427 & & & $3.71 \mathrm{E}-03$ & 99A1-99A1 \\
\hline CG10512 & FBgn0037057 & & & $3.80 \mathrm{E}-03$ & 78C2-78C2 \\
\hline Troponin C 41F & FBgn0033027 & & & $4.79 \mathrm{E}-03$ & $41 \mathrm{~F} 2-41 \mathrm{~F} 3$ \\
\hline Ribosomal protein S18 & FBgn0010411 & & & 4.87E-03 & 56F11-56F11 \\
\hline Histone $\mathrm{H} 4$ replacement & FBgn0013981 & & & $5.00 \mathrm{E}-03$ & 88C9-88C9 \\
\hline Malic enzyme & FBgn0002719 & & & $5.14 \mathrm{E}-03$ & 87C6-87C7 \\
\hline Cytochrome $c$ oxidase $\mathrm{Va}, \mathrm{mt}$ precursor & FBgn0019624 & ox & $\mathrm{m}$ & $5.21 \mathrm{E}-03$ & 86F9-86F9 \\
\hline Ribosomal protein L12 & FBgn0034968 & & & $5.88 \mathrm{E}-03$ & 60B7-60B7 \\
\hline CG16712 & FBgn0031561 & & & $6.70 \mathrm{E}-03$ & $24 \mathrm{~B} 3-24 \mathrm{~B} 3$ \\
\hline CG33138 & FBgn0053138 & & & $7.14 \mathrm{E}-03$ & 49F4-49F4 \\
\hline Stress-inducible humoral factor Turandot C & FBgn0044812 & & & $7.44 \mathrm{E}-03$ & $93 \mathrm{~A} 2-93 \mathrm{~A} 2$ \\
\hline ATP synthase B chain, mt precursor & FBgn0019644 & ox & $\mathrm{m}$ & $7.63 \mathrm{E}-03$ & 67C5-67C5 \\
\hline CG8343 & FBgn0040502 & & & $7.66 \mathrm{E}-03$ & $42 \mathrm{~A} 13-42 \mathrm{~A} 13$ \\
\hline
\end{tabular}

Abbreviations: ATP, adenosine triphosphate; QTL, quantitative trait locus.

The $P$-value from $t$-test, FlyBase accession number and cytological position are listed. Proteins in boldface are proteins located within the QTL region. Proteins associated with oxidative phosphorylation (ox) and mitochondria $(\mathrm{m})$ are indicated based on the gene-enrichment-analysis. 


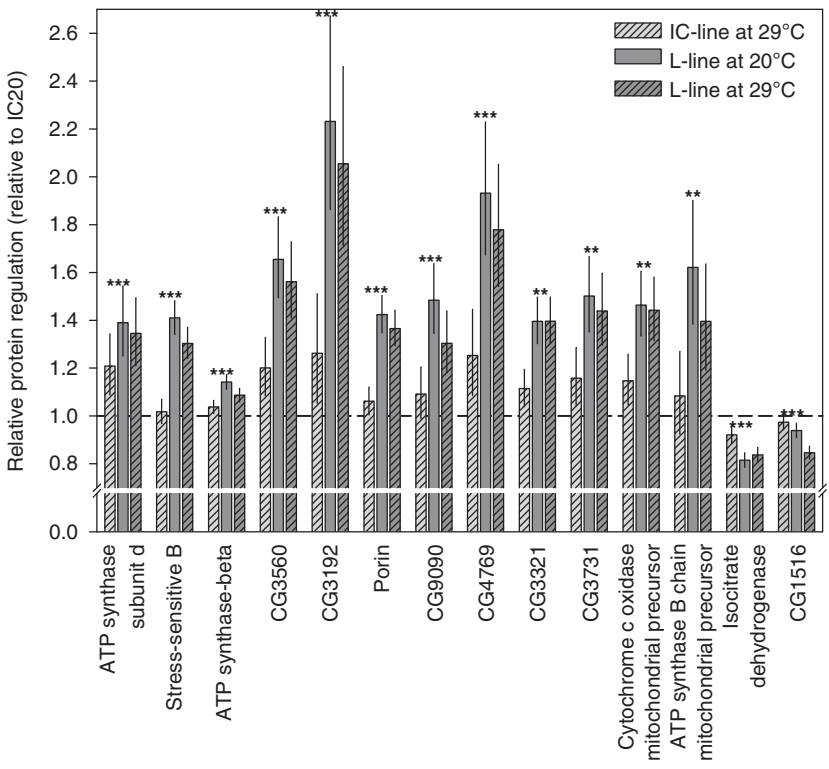

Figure 3 Regulation of proteins associated with the mitochondria and oxidative phosphorylation (mean \pm error factor) based on the database for annotation, visualization and integrated discovery (DAVID) results. Quantification for each protein at the different temperatures is relative to the inbred-control-line (IC-line) at the permissive temperature $\left(20^{\circ} \mathrm{C}\right)$. Level of IC-line at the permissive temperature is indicated by the horizontal dashed line. The asterisks indicate significance level for differential regulation in response to the restrictive temperature $\left({ }^{* *} P<0.01 ;{ }^{* * *} P<0.001\right)$.

cantly differently regulated between the IC-line and the L-line are listed in Table 1. Previous identification of a QTL region harboring the causal mutation allows separation of causation and secondary consequences. Thus, cytological position of genes coding for proteins in Table 1 are also listed, and genes mapping to the QTL region are highlighted in bold. The two proteins, (1) voltage-dependent anion-selective channel (porin) and (2) Adh-related, are coded by genes locating to the identified QTL region (Vermeulen et al., 2008). Porin shows a significantly higher expression level in the L-line in comparison with the IC-line at both temperature conditions, and is regulated in the opposite direction in response to the restrictive temperature (Figure 3 ). The protein, stress-sensitive $B$, which is a mitochondrial adenine nucleotide translocase, was also observed to be overexpressed in the L-line at both temperatures (Figure 3). In the IC-line, stress-sensitive B is not regulated in response to the restrictive temperature, but in the L-line it is observed to be downregulated in response to the restrictive temperature (Figure 3). Expression levels of a subset of the differentially regulated proteins in the L-line are presented in Figure 4. Several proteins related to muscle contraction show a differential regulation pattern in the L-line in comparison with the IC-line (Figure 4). Two stress-responsive proteins (Turandot $\mathrm{A}$ and $\mathrm{C}$ ) show exceptionally distinct expression levels in the L-line (Figure 4). Both Turandot proteins are expressed at very low levels in the L-line and, most notably, do not show as large a response to the restrictive temperature as the IC-line. A full list of all relative ratios, error factors and the number of peptides identifying each protein is given in Supplementary Table S3. No significant differences in the proteomic response

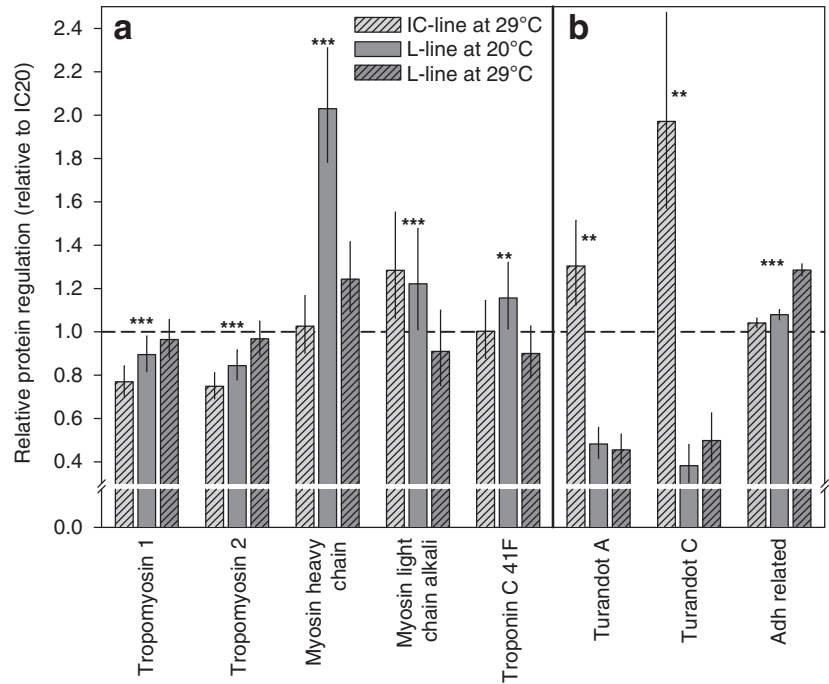

Figure 4 (a) Regulation of differentially regulated proteins (mean \pm error factor) related to muscle contraction. (b) Regulation of some of the other differentially regulated proteins (mean \pm error factor). Quantification for each protein at the different temperatures is relative to the inbred-control-line (IC-line) at the permissive temperature $\left(20^{\circ} \mathrm{C}\right)$. Level of IC-line at the permissive temperature is indicated by the horizontal line. The asterisks indicate significance level for differential regulation in response to the restrictive temperature $\left.{ }^{* *} P<0.01 ;{ }^{* * *} P<0.001\right)$.

to the restrictive temperature were found when comparing the two control lines (IC-line and OC-line) in experiment 2 (results not shown). This indicates that the differently regulated proteins found in the L-line are not due to effects created by inbreeding of the IC-line, and that the regulation patterns specific to the L-line are associated with the extreme mortality at the restrictive temperature. The significantly higher expression level of porin in the L-line was verified in experiment 3 (Supplementary Figure S3), indicating that porin has a central role in the conditional mortality of the L-line. The regulation pattern of Adh-related distinct to the L-line was also retrieved in experiment 3 (Supplementary Figure S3).

To test whether there was an overrepresentation of proteins within specific processes and pathways, the list of proteins identified to have a unique regulation in the L-line (Table 1) was subjected to gene-enrichmentanalysis using the database for annotation, visualization and integrated discovery functional annotation tool (DAVID) (Dennis et al., 2003; Hosack et al., 2003). To account for overrepresentation of proteins within specific pathways due to biased protein identification, all proteins identified in experiment 1 were used as a background search list. A gene-enrichment-analysis evaluates the probability of detecting the observed number of genes within a category by evaluating the proportion of observed genes in the gene list belonging to a category versus the proportion of all the genes in the background search list. Significance criteria for the gene-enrichmentanalysis were (1) more than three genes within each category and (2) an EASE-score $P$-value $<0.05$, and all category specificity levels of gene-ontology themes are listed. Note that the hierarchical construction of geneontology themes allows genes to be present in several 
Table 2 Gene-enrichment-analysis of significantly differently regulated proteins (from Table 1)

\begin{tabular}{|c|c|c|c|}
\hline & No. of genes & Percentage of group & EASE score P-value \\
\hline \multicolumn{4}{|l|}{ Biological process } \\
\hline Generation of precursor metabolites and energy & 15 & 31.9 & $6.40 \mathrm{E}-03$ \\
\hline Ion transport & 8 & 17.0 & $8.40 \mathrm{E}-03$ \\
\hline Oxidative phosphorylation & 9 & 19.1 & $2.80 \mathrm{E}-02$ \\
\hline \multicolumn{4}{|l|}{ Molecular function } \\
\hline Transmembrane transporter activity & 11 & 23.4 & $8.00 \mathrm{E}-04$ \\
\hline Ion transmembrane transporter activity & 10 & 21.3 & $1.90 \mathrm{E}-03$ \\
\hline Substrate-specific transmembrane transporter activity & 10 & 21.3 & $2.30 \mathrm{E}-03$ \\
\hline Transporter activity & 11 & 23.4 & $5.60 \mathrm{E}-03$ \\
\hline Substrate-specific transporter activity & 10 & 21.3 & $8.70 \mathrm{E}-03$ \\
\hline Inorganic cation transmembrane transporter activity & 8 & 17.0 & $2.00 \mathrm{E}-02$ \\
\hline Cation transmembrane transporter activity & 8 & 17.0 & $2.00 \mathrm{E}-02$ \\
\hline Hydrogen ion transmembrane transporter activity & 7 & 14.9 & $4.10 \mathrm{E}-02$ \\
\hline Monovalent inorganic cation transmembrane transporter activity & 7 & 14.9 & $4.80 \mathrm{E}-02$ \\
\hline \multicolumn{4}{|l|}{ Cellular compartment } \\
\hline Mitochondrial envelope & 12 & 25.5 & $1.30 \mathrm{E}-04$ \\
\hline Envelope & 12 & 25.5 & $1.70 \mathrm{E}-04$ \\
\hline Organelle envelope & 12 & 25.5 & $1.70 \mathrm{E}-04$ \\
\hline Mitochondrial membrane & 11 & 23.4 & $4.60 \mathrm{E}-04$ \\
\hline Mitochondrial inner membrane & 10 & 21.3 & $1.90 \mathrm{E}-03$ \\
\hline Organelle inner membrane & 10 & 21.3 & $1.90 \mathrm{E}-03$ \\
\hline Organelle membrane & 11 & 23.4 & $2.10 \mathrm{E}-03$ \\
\hline Mitochondrial membrane part & 9 & 19.1 & $4.80 \mathrm{E}-03$ \\
\hline Mitochondrial part & 13 & 27.7 & $7.30 \mathrm{E}-03$ \\
\hline Membrane part & 11 & 23.4 & $8.10 \mathrm{E}-03$ \\
\hline Membrane & 12 & 25.5 & $9.60 \mathrm{E}-03$ \\
\hline Mitochondrion & 14 & 29.8 & $3.50 \mathrm{E}-02$ \\
\hline
\end{tabular}

The table shows the number of genes identified within specific groups, the percentage of total genes within groups and the EASE score $P$-value for the Gene Ontology Themes 'biological process', 'molecular function' and 'cellular compartment'.

groups and more specific categories to be part of less specific categories (Dennis et al., 2003). Results from the gene-enrichment-analysis are presented in Table 2. In particular, a significant overrepresentation of proteins involved in oxidative phosphorylation (ox; Table 1), and associated with the mitochondrial cellular compartment (m; Table 1) was observed. Regulation of proteins related to oxidative phosphorylation and the mitochondria is presented in Figure 3. The majority of proteins related to mitochondrial processes are overexpressed in the L-line. Proteins associated with ion transport and generation of precursor metabolites and energy were also significantly overrepresented within the list of differentially regulated proteins. The significantly overrepresented processes and functions point to a dysfunctional regulation of mitochondrial function in the L-line in response to the restrictive temperature. The significantly differently expressed proteins identified in experiment 3 between the L-line and IC-line at the permissive temperature and at the restrictive temperature (Supplementary Tables S4 and S5) were also subjected to gene-enrichment-analysis. All identified proteins in experiment 3 were used as background search list and the significance criteria were the same as for experiment 1. Results from the gene-enrichment-analysis are listed in Supplementary Tables S1 and S2. In addition, in this experiment the significant geneontology categories differentiating the L-line and IC-line are, for example, related to mitochondrial processes, transporter activity, oxireductase activity and membranebound processes. These results support that processes associated with mitochondrial function and adenosine triphosphate (ATP) production are affected by the lethal mutation in the L-line at the restrictive temperature.

\section{Discussion}

Environmentally induced expression of genetic variation is a topic of high relevance within diverse research areas, such as medicine, evolutionary biology and population genetics (Rutherford and Lindquist, 1998; Willis et al., 2006; Peng et al., 2007). More specifically, analyses on the genetic load that are conditioned by the environment are of utmost importance for characterizing and identifying causations of inbreeding by environment interactions (Kristensen et al., 2008; Vermeulen et al., 2008). The deleterious effects of inbreeding are generally accepted to be more adverse in suboptimal environments (Bijlsma et al., 2000; Armbruster and Reed, 2005), but knowledge of the environmentally induced causative molecular processes are scarce. In addition, molecular functional characterization of inbreeding-related phenomena may contribute to resolve the long-standing debate about the relative contributions of major genes to inbreeding depression. Recent evidence suggests that inbreeding depression is caused by a few major genes that in turn affect many other genes (Ayroles et al., 2009). Analyses of temperature-sensitive mutations are of relevance in relation to predicting the effect of global warming, besides also being important in relation to inbreeding by environment interactions (Parmesan and Yohe, 2003). Characterization of the pathways and processes showing sensitivity to temperature change can contribute to an improved understanding of physiological limitations of adaptations to temperature change.

We present a proteomic characterization of a naturally occurring conditional lethal mutation found in an inbred line of $D$. melanogaster. In all, 45 proteins were 
found to be differentially regulated in the L-line as compared with the IC-line in response to the restrictive temperature $\left(29^{\circ} \mathrm{C}\right)$. No differences in the proteomic response to the restrictive temperature were identified between the IC-line and the OC-line, verifying that differential protein regulation in the L-line is not caused by general inbreeding effects (Kristensen et al., 2005). Proteome analysis of differential regulation patterns between the L-line and the IC-line in response to the restrictive temperature was repeated 40 generations later to verify the distinct proteomic responses observed in the L-line. Proteins differentially regulated in response to the restrictive temperature are presumably related to the large increase in mortality observed in the L-line in response to the restrictive temperature. The identified QTL region can be used to give an indication whether the distinct protein regulation patterns identified in the L-line are causative or represent secondary cellular effects.

\section{Mitochondrial-related processes}

Within the list of proteins responding distinctively in the L-line, a significant overrepresentation of proteins implicated in oxidative phosphorylation and associated with mitochondrial functions were observed. This indicates that fundamental processes related to mitochondrial function, perhaps specifically ATP synthesis, are distorted in L-line flies when exposed to the restrictive temperature. The majority of proteins associated with oxidative phosphorylation and mitochondrial function are overexpressed in the L-line (Figure 3). The markedly elevated expression of mitochondrial proteins suggests that suboptimal mitochondrial function in L-line flies are compensated by overexpression of the entire mitochondrial machinery. The protein, stress-sensitive B, which is a mitochondrial ATP/ADP translocase transferring ATP into the cytoplasm in exchange for ADP, was observed at higher expression levels in the L-line (Figure 3). A point mutation in stress-sensitive $B$ has previously been shown to be related to temperature sensitivity, resulting in a paralytic behavior at increased temperatures (Rikhy et al., 2003). In essence, this suggests that dysfunction of mitochondrial function is a major factor in the high mortality observed in the L-line. Other analyses on the molecular consequences of environmental stress, inbreeding and aging have also observed genes related to energy metabolism to be affected (Landis et al., 2004; Kristensen et al., 2006; Sørensen et al., 2007). This indicates that energy metabolism is a highly sensitive and fine-tuned process that can respond to a diverse range of stimuli. However, fine-tuned processes are also especially vulnerable to expression of deleterious alleles. The most common cause for temperature sensitivity is suggested to be amino acid changes affecting activity and stability of proteins, which will severely affect efficiency of energy metabolism (Hochachka and Somero, 1968; Yampolsky and Stoltzfus, 2005).

The two differentially regulated proteins, Adh-related and porin, are coded by genes located in the identified QTL region, known to segregate variation affecting expression of the lethal effect. The large number of differentially regulated proteins not located in the QTL region indicates a high level of secondary effects caused by epistatic interaction between genes. Molecular secondary consequences may be observed either because of the inefficiency of specific steps in a pathway, thus affecting following steps in the pathway or as a compensatory process to stabilize the genotype to phenotype translation. Large secondary effects caused by interaction between genes have previously been observed on the gene expression level (Ayroles et al., 2009). It should be noted that expression analyses are highly suitable to characterize molecular phenotypes, but rarely have the resolution to unambiguously pinpoint the causal gene. Supplementary analyses that can potentially lead to the identification of the causal mutation and further characterize the molecular processes underlying the mortality in the L-line are discussed in the next section. Nonetheless, the regulation pattern of the voltage-dependent anion-selective channel protein, Porin, is interesting because it is closely associated with mitochondrial function. Ion channel kinetics in $D$. melanogaster have previously been shown to be sensitive to elevated temperatures (Peng et al., 2007), thereby distorting physiological and developmental processes essential for cellular functions. Porin is a pore-forming protein situated in the outer mitochondrial membrane having a central role in movement of metabolites across the outer membrane, and is known to affect energy metabolism and apoptosis (Komarov et al., 2004; Abu-Hamad et al., 2006; Lee et al., 2007). Previously, a recessive mutation in the porin gene has been shown to cause lethality or elevated early mortality and to be expressed in a sex-specific pattern (Oliva et al., 2002; Lee et al., 2007). Sixteen potential phosphorylation sites have been identified on the $D$. melanogaster porin protein, indicating a very fine-tuned regulation by posttranslational phosphorylation (Pical et al., 1993; Lee et al., 2007). This may imply increased mutational constrains, and as a result optimal function of porin may be highly vulnerable.

Phenotypic observations indicate that L-line flies show hyper-contraction in the moribund phase, which is in accordance with our findings of several differentially regulated proteins involved in muscle contraction (Figure 4). Hyper-contraction can be a secondary effect, unleashed by dysfunctional ATP synthesis and regulation. The differentially regulated proteins, porin and stress-sensitive B, which are both associated with mitochondrial function, are known to interact with a capping protein $(c p b)$ involved in actin binding and actin filament organization (Giot et al., 2003; Wear and Cooper, 2004; Janody and Treisman, 2006). In addition, the results indicate that porin is the binding site for hexokinase and thereby facilitates the access of kinases to ATP in oxidative muscle (Anflous-Pharaya et al., 2007). Porindeficient mutant mice (double voltage-dependent anion channel mutants) show a markedly reduced capacity for exercise, corroborating that porin is essential for oxidative phosphorylation and ATP synthesis (Anflous-Pharaya et al., 2007). The essential role of porin in cellular survival and energy metabolism makes it an interesting starting point of analyses of the temperature-specific mortality of the L-line.

\section{Towards the identification of the causal mutation}

Genetic dissection and characterization of phenotypic variation is not as trivial as sometimes believed 
(Andersson, 2001). However, our results significantly narrow down the number of genes and pathways to analyze in more detail, and thus present a step on the way to identify and understand phenotypic consequences of expression of the causal mutation. Several different approaches analyzing effects on diverse biological levels can be used in addition to the knowledge obtained until now about the lethal effect in this inbred line. To further narrow down the genetic region harboring the causal mutation, fine-scale deficiency mapping using molecularly mapped deletions in the QTL region are in progress. Additional analysis of genome-wide gene expression, using the same lines and experimental design as used for the proteome analysis presented here, is also in progress. Integrated analysis and interpretation of all acquired data (QTL region, fine-scale mapping, transcriptome, proteome and phenotypic data) will further narrow down the region, and very likely pinpoint the gene in which the causal mutation is located, and present a functional characterization of the dysfunctional processes causing extremely high mortality at restrictive conditions in the L-line. A combination of the QTL and proteome expression results can provide insight into the extent of epistatic interactions between genes, geneenvironment interactions and how the genotype translates into the phenotype.

The quantitative proteomic approach used in this study to analyze the environmentally induced mortality of the L-line is a highly robust and sensitive technology; however, it is a non-targeted explorative approach. This kind of study presents an overall picture of the accessible proteome using this technology and can provide the first insight into the functional processes disturbed in the L-line. Targeted proteomic approaches (selected reaction monitoring) in which specific proteins or pathways are monitored are just recently appearing (Lange et al., 2008). To further characterize the functional causation of the mortality in the L-line, a targeted proteomic approach would be suitable. Our data indicate that processes related to oxidative phosphorylation and mitochondria should be analyzed in more detail in a targeted proteomic approach. Another interesting approach would be to include all proteins coded by genes located in the QTL region, or genes related to mitochondrial function located in the QTL region, in a targeted proteome analysis; this could also provide data with the potential to identify the causal mutation. An obvious next step in locating the causal mutation is to perform comparative sequencing of the full QTL region, searching for fixed homozygous differences between the L-line and several other outbred and inbred lines. Nevertheless, even though sequencing techniques are becoming increasingly fast and economically accessible, this would still be an immense task, considering the length of the QTL region and the number of individuals necessary to sequence.

\section{Conclusion}

Our study is among the first to characterize protein expression patterns related to conditional deleterious effects of inbreeding. Overall, our results indicate that the high early mortality of L-line flies at the restrictive temperature is associated with a change of proteins related to oxidative phosphorylation and/or other mitochondrial processes. The phenotypic observation of abnormal muscle hyper-contraction in the moribund phase of the L-line corroborates that mitochondrial processes and/or ATP turnover have a key role in L-line mortality at the restrictive temperature. Matching of proteome expression patterns to the QTL region offers a unique opportunity to separate causal effects and secondary consequences that has shown to be highly relevant in analyzing the molecular basis of inbreeding depression. Our results indicate that expression of simple genetic defects can induce large secondary responses. Besides providing knowledge of temperature sensitivity, characterization of a conditionally expressed mitochondrial dysfunction in a tractable model organism, as D. melanogaster, has the potential to reveal insights into mitochondrial function and defects that are otherwise difficult to study.

\section{Acknowledgements}

We are grateful to Torsten Nygaard Kristensen, Ian Max Møller, Marianne Danielsen and Kuke Bijlsma for their valuable comments and discussions on the paper. This work was supported by the Graduate School of Environmental Stress Studies (KSP), Villum Kann Rasmussen Foundation (CV) and the Danish Natural Sciences Research Council (VL).

\section{References}

Abu-Hamad S, Sivan S, Shoshan-Barmatz V (2006). The expression level of the voltage-dependent anion channel controls life and death of the cell. Proc Natl Acad Sci USA 103: 5787-5792.

Andersson L (2001). Genetic dissection of phenotypic diversity in farm animals. Nat Rev Genet 2: 130-138.

Anflous-Pharaya K, Cai Z-J, Craigen WJ (2007). VDAC1 serves as a mitochondrial binding site for hexokinase in oxidative muscles. Biochim Biophys Acta 1767: 136-142.

Arking R (1975). Temperature sensitive cell-lethal mutants of Drosophila: isolation and characterization. Genetics 80: 519-537.

Armbruster P, Reed DH (2005). Inbreeding depression in benign and stressful environments. Heredity 95: 235-242.

Ayroles JF, Hughes KA, Rowe KC, Reedy MM, Rodriguez-Zas SL, Drnevich JM et al. (2009). A genomewide assessment of inbreeding depression: gene number, function and mode of action. Conserv Biol 23: 920-930.

Bijlsma R, Bundgaard J, Boerema AC (2000). Does inbreeding affect the extinction risk of small populations?: predictions from Drosophila. J Evol Biol 13: 502-514.

Biron DG, Loxdale HD, Ponton F, Moura H, Marche L, Brugidou C et al. (2006). Population proteomics: an emerging discipline to study metapopulation ecology. Proteomics 6: 1712-1715.

Bobula J, Tomala K, Jez E, Wloch DM, Borts RH, Korona R (2006). Why molecular chaperones buffer mutational damage: a case study with a yeast Hsp40/70 system. Genetics 174: 937-944.

Charlesworth B, Charlesworth D (1999). The genetic basis of inbreeding depression. Genet Res 74: 329-340.

Chevalier F, Martin O, Rofidal V, Devauchelle A-D, Barteau S, Sommerer $\mathrm{N}$ et al. (2004). Proteomic analyzation of natural variation between Arabidopsis ecotypes. Proteomics 4: 1372-1381.

Chong PK, Gan CS, Pham TK, Wright PC (2006). Isobaric tags for relative and absolute quantitation (iTRAQ) reproduci- 
bility: implication of multiple injections. J Proteome Res 5: 1232-123. 1240.

Danielsen M, Hornshøj H, Siggers RH, Jensen BB, van Kessel AG, Bendixen E (2007). Effects of bacterial colonization on the porcine intestinal proteome. J Proteome Res 6: 2596-2604.

Dennis JG, Sherman BT, Hosack DA, Yang J, Gao W (2003). DAVID: database for annotation, visualization, and integrated discovery. Genome Biol 4: R60.

Gan CS, Chong PK, Pham TK, Wright PC (2007). Technical, experimental, and biological variations in isobaric tags for relative and absolute quantitation (iTRAQ). J Proteome Res 6: 821-827.

Giot L, Bader JS, Brouwer C, Chaudhuri A, Kuang B, Li Y et al. (2003). A protein interaction map of Drosophila melanogaster. Science 302: 1727-1736.

Hochachka PW, Somero GN (1968). The adaptation of enzymes to temperature. Comp Biochem Physiol 27: 659-668.

Homyk JT, Sinclair DAR, Wong DTL, Grigliatti TA (1986). Recovery and characterization of temperature-sensitive mutations affecting adult viability in Drosophila melanogaster. Genetics 113: 367-389.

Hosack DA, Dennis JG, Sherman BT, Lane HC, Lempicki RA (2003). Identifying biological themes within lists of genes with EASE. Genome Biol 4: R70.

Janody F, Treisman JE (2006). Actin capping protein $\alpha$ maintains vestigial-expressing cells within the Drosophila wing disc epithelium. Development 133: 3349-3357.

Jedlicka P, Mortin MA, Wu C (1997). Multiple functions of Drosophila heat shock transcription factor in vivo. EMBO J 16: 2452-2462.

Karr TL (2008). Application of proteomics to ecology and population biology. Heredity 100: 200-206.

Komarov AG, Graham BH, Craigen WJ, Colombini M (2004). The physiological properties of a novel family of VDAC-like proteins from Drosophila melanogaster. Biophys J 86: 152-162.

Kristensen TN, Barker JSF, Pedersen KS, Loeschcke V (2008). Extreme temperatures increase the deleterious consequences of inbreeding under laboratory and semi-natural conditions. Proc Roy Soc B Biol Sci 275: 2055-2061.

Kristensen TN, Sørensen P, Kruhøffer M, Pedersen KS, Loeschcke V (2005). Genome-wide analysis on inbreeding effects on gene expression in Drosophila melanogaster. Genetics 171: $157-167$.

Kristensen TN, Sørensen P, Pedersen KS, Kruhøffer M, Loeschcke V (2006). Inbreeding by environment interactions affect gene expression in Drosophila melanogaster. Genetics 173: 1329-1336.

Landis GN, Abdueva D, Skvortsov D, Yang J, Rabin BE, Carrick $J$ et al. (2004). Similar gene expression patterns characterize aging and oxidative stress in Drosophila melanogaster. Proc Natl Acad Sci USA 101: 7663-7668.

Lange V, Picotti P, Domon B, Aebersold R (2008). Selected reaction monitoring for quantitative proteomics: A tutorial. Mol Syst Biol 4: 222

Lee S, Leung HT, Kim E, Jang J, Lee E, Baek K et al. (2007). Effects of a mutation in the Drosophila porin gene encoding mitochondrial voltage-dependent anion channel protein on phototransduction. Dev Neurobiol 67: 1533-1545.

Maisnier-Patin S, Roth JR, Fredriksson $\AA$, Nyström T, Berg OG Andersson DI (2005). Genomic buffering mitigates the effects of deleterious mutations in bacteria. Nat Genet 37: 1376-1379.

Mayoh H, Suzuki DT (1973). Temperature-sensitive mutations in Drosophila melanogaster. 16. Genetic properties of sexlinked recessive cold-sensitive mutants. Can J Genet Cytol 15: 237-254.

Mitchell HK, Petersen NC (1982). Developmental abnormalities in Drosophila induced by heat shock. Dev Genet 3: 91-102.
Oliva M, De Pinto V, Barsanti P, Caggese C (2002). A genetic analysis of the porin gene encoding a voltage-dependent anion channel protein in Drosophila melanogaster. Mol Genet Genomics 267: 746-756.

Parmesan C, Yohe G (2003). A globally coherent fingerprint of climate change impacts across natural systems. Nature $\mathbf{4 2 1}$ : 37-42.

Peng IF, Berke BA, Zhu Y, Lee WH, Chen WJ, Wu CF (2007). Temperature-dependent developmental plasticity of Drosophila neurons: cell-autonomous roles of membrane excitability, $\mathrm{Ca}^{2+}$ influx, and cAMP signaling. J Neuroci 27 : 12611-12622.

Pical C, Fredlund KM, Petit PX, Sommarin M, Møller IM (1993). The outer membrane of plant mitochondria contains a calcium-dependent protein kinase and multiple phosphoproteins. FEBS Lett 336: 347-351.

Rikhy R, Ramaswami M, Krishnan KS (2003). A temperaturesensitive allele of Drosophila ses $B$ reveals acute functions for the mitochondrial adenine nucleotide translocase in synaptic transmission and dynamin regulation. Genetics 165: 1243-1253.

Rutherford SL, Lindquist S (1998). Hsp90 as a capacitor for morphological evolution. Nature 396: 336-342.

Shilov IV, Seymour SL, Patel AA, Loboda A, Tang WH, Keating SP et al. (2007). The Paragon Algorithm, a next generation search engine that uses sequence temperature values and feature probabilities to identify peptides from tandem mass spectra. Moll Cell Proteomics 6: 1638-1655.

Storey JD (2007). The optimal discovery procedure: a new approach to simultaneous significance testing. J Roy Stat Soc B 69: 347-368.

Suzuki DT (1970). Temperature-sensitive mutations in Drosophila melanogaster. Science 170: 695-706.

Sørensen JG, Nielsen MM, Loeschcke V (2007). Gene expression profile analysis of Drosophila melanogaster selected for resistance to environmental stress. J Evol Biol 20: 1624-1636.

Tasaka SE, Suzuki DT (1973). Temperature sensitive mutations in Drosophila melanogaster XVII heat- and cold-sensitive lethals on chromosome 3. Genetics 74: 509-520.

Thiellement H, Zivy M, Plomion C (2002). Combining proteomic and genetic studies in plants. J Chromatogr B 782: 137-149.

Thomsen B, Horn P, Panitz F, Bendixen E, Petersen AH, Holm LE et al. (2006). A missense mutation in the bovine SCL35A3 gene, encoding a UDP-N-acetylglucosamine transporter, causes complex vertebral malformation. Genome Res 16: 97-105.

Vermeulen CJ, Bijlsma R (2004a). Changes in mortality patterns and temperature dependence of lifespan in Drosophila melanogaster caused by inbreeding. Heredity 92: 275-281.

Vermeulen CJ, Bijlsma R (2004b). Characterization of conditionally expressed mutants affecting age-specific survival in inbred lines of Drosophila melanogaster: lethal conditions and temperature sensitive periods. Genetics 167: 1241-1248.

Vermeulen CJ, Bijlsma R, Loeschcke V (2008). A major QTL affects temperature sensitive adult lethality and inbreeding depression in life span in Drosophila melanogaster. BMC Evol Biol 8: 297.

Wear MA, Cooper JA (2004). Capping protein: new insights into mechanism and regulation. Trends Biochem Sci 29: 418-428.

Willis JH, Munro E, Lyczak R, Bowerman B (2006). Conditional dominant mutations in the Caenorhabditis elegans gene act-2 identify cytoplasmic and muscle roles for a redundant actin isoform. Mol Biol Cell 17: 1051-1064.

Yampolsky LY, Stoltzfus A (2005). The exchangeability of amino acids in proteins. Genetics 170: 1459-1472. 